\title{
Feasibility and safety of angiotensin II administration in general ward patients during COVID-19 pandemic: a case series
}

Federica Morselli, Giordano Vitali, Elena Brioschi, Gaetano Di Terlizzi, Alessandro Belletti, Gaetano Lombardi, Giacomo Senarighi, Ary Serpa Neto, Alberto Zangrillo and Giovanni Landoni

www.doi.org/10.51893/2020.4.rl1

Published online first 7 December 2020

From December 2019, the severe acute respiratory syndrome coronavirus 2 (SARS-CoV-2) has caused a pandemic of coronavirus disease 2019 (COVID-19) that has frequently overwhelmed health care systems due to the high number of patients requiring admission to an intensive care unit (ICU). 1,2

Angiotensin-converting enzyme 2 (ACE2) is a cellular membrane-bound vasopeptidase that has been identified as a functional receptor for SARS-CoV-2. ${ }^{3}$

Angiotensin II is an endogenous octapeptide with vasoconstrictor activity that has been recently suggested as a catecholamine-sparing agent for vasodilatory shock. ${ }^{4}$ It has been hypothesised that angiotensin II may act as competitive inhibitor for ACE2 with SARS-CoV-2 and may therefore have antiviral activity. ${ }^{5}$

We have previously described our experience with the use of intravenous angiotensin II as compassionate therapy for patients with COVID-19 admitted to the ICU.6,7 Early antiviral treatment may be associated with greater benefits. Therefore, we started administering intravenous angiotensin II as antiviral to patients with moderate COVID19-related acute respiratory distress syndrome (ARDS) in the general ward, before invasive ventilation or shock requiring ICU admission. No published data are available on use of intravenous angiotensin II outside the controlled environment of the ICU. In this article, we provide our data on feasibility and safety of angiotensin II administration in a non-ICU setting.

We present a case series of seven COVID-19 patients with moderate ARDS who received intravenous angiotensin II in the general ward. Intravenous use of angiotensin II is not approved by the Italian Drug Administration Agency (Agenzia Italiana del Farmaco [AIFA]) or the European Medicines Agency (EMA). Accordingly, we obtained ethics approval for compassionate use of the drug and all patients provided a written informed consent. The data collection was part of the COVID-BioB study approved by the Hospital Ethics Committee (Protocol No. 34/int/2020) and registered on ClinicalTrials.gov (NCT04318366). Hospital organisation and clinical management of patients with COVID-19 at our centre have been previously published. 1,6

We included patients who had confirmed SARS-CoV-2 infection with clinical or radiological signs of interstitial pneumonia and moderate ARDS.

Patients with cardiogenic shock, stage IV cancer, contraindications to deep venous thrombosis prophylaxis, hypertension requiring treatment, or receiving invasive ventilation at the moment of enrolment were excluded.

Details on patient monitoring, study drug preparation and administration, data collection, and study outcomes are presented in the Online Appendix. Angiotensin II (drug concentration $25 \mu \mathrm{g} / \mathrm{mL}$ ) was infused in a dedicated vein at a starting dose of $5 \mathrm{ng} / \mathrm{kg} / \mathrm{min}$, aiming to achieve a mean arterial pressure greater than $65 \mathrm{mmHg}$ unless clinical judgement suggested targeting higher values (eg, reduction in urinary output). If a dose of $10 \mathrm{ng} / \mathrm{kg} / \mathrm{min}$ or over was reached, the patient was evaluated for vasopressor support and ICU admission.

Data were collected from medical records by trained investigators not involved in patient management. Haemodynamic and ventilatory variables were collected before angiotensin II administration and at 1, 2, 4, 6, 12, 24 and 48 hours after initiating treatment with angiotensin II. Patients were followed up until day 28.

The primary outcome was clinical improvement at day 28 - defined as an improvement of 2 points or more on a six-category ordinal scale compared with baseline. ${ }^{8}$ Secondary outcomes included mortality, need for ICU admission and need for invasive mechanical ventilation. Safety outcomes were drug extravasation, limb or bowel ischaemia, arrhythmias, or uncontrolled hypertension during angiotensin II administration.

Baseline characteristics are presented in the Online Appendix (table 1). All patients had moderate ARDS at time of enrolment. Non-invasive ventilation was administered with $10 \mathrm{mmHg}$ positive end-expiratory pressure in all patients, and $\mathrm{PaO}_{2} / \mathrm{FiO}_{2}$ (arterial partial pressure of oxygen/fraction of 


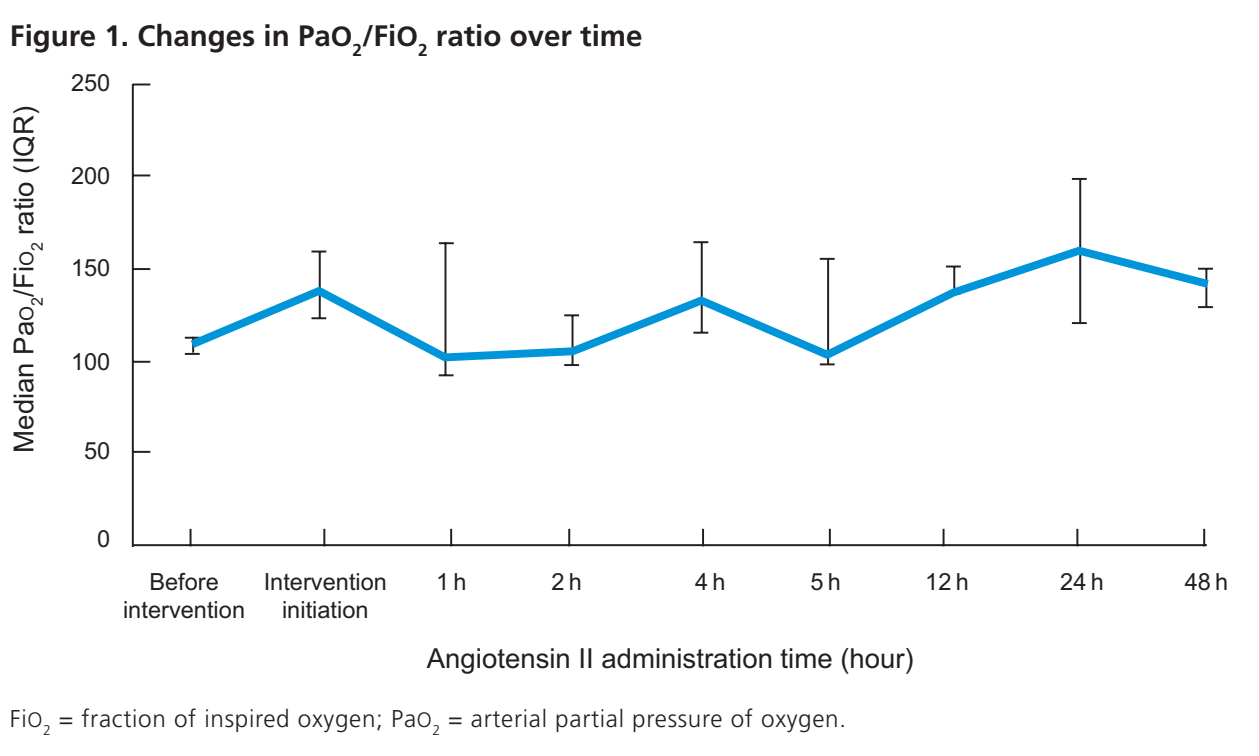

two of whom died. In both cases, the main cause of death was barotrauma (pneumomediastinum). At day 28, one patient remained in the ICU, but was discharged after day 28 , and all other patients were discharged from hospital (Table 1). No patient developed complications related to angiotensin ॥ administration. In particular, there were no cases of drug extravasation, limb or bowel ischaemia, arrhythmias, or uncontrolled hypertension. After clinical improvement, a mild cutaneous hand rash was noted in a patient and angiotensin II was interrupted on a precautionary basis.

In this study, to our knowledge, we present for the firsttime clinical data on the feasibility and safety of angiotensin II administration in hospital wards. Notably, our hospital already has longstanding experience in non-invasive ventilation and vasopressor administration outside the ICU. 2,9 In our published experience, we treated 129 patients with non-invasive ventilation on the ward over 7 months (about one new patient every 2 days). In our non-published recent experience, we have on average four hospitalised patients receiving vasopressors outside the ICU. Our study suggests that vasopressor administration outside the ICU is feasible, and may be considered in low resource settings such as during pandemics or major emergencies. Furthermore, our study is reassuring that low dose vasopressor on ward administration may be considered as a method to reduce the need for ICU beds and improve resource allocation. ${ }^{10}$

Nevertheless, larger studies are needed to ultimately confirm safety, efficacy and cost-effectiveness of angiotensin II administration in wards.

Acknowledgements: We thank all the personnel of San Raffaele Hospital for the dedication to these patients and for the support in data collection. We acknowledge our collaborators from IRCCS San Raffaele Scientific Institute, Milan, Italy: Gabriele Valsecchi, Gabriele Todaro, Monica Fedrizzi, Francesca Conte and Davide Losi.

\section{Competing interests}

None declared. 


\section{RESEARCH LETTERS}

\section{Author details}

Federica Morselli ${ }^{1}$

Giordano Vitali ${ }^{1}$

Elena Brioschi ${ }^{1}$

Gaetano Di Terlizzi

Alessandro Belletti ${ }^{1}$

Gaetano Lombardi ${ }^{1}$

Giacomo Senarighi'

Ary Serpa Neto ${ }^{2,3}$

Alberto Zangrillo ${ }^{1,4}$

Giovanni Landoni ${ }^{1,4}$

1 IRCCS San Raffaele Scientific Institute, Milan, Italy.

2 Australian and New Zealand Intensive Care Research Centre (ANZIC-RC), School of Public Health and Preventive Medicine, Monash University, Melbourne, VIC, Australia.

3 Department of Critical Care Medicine, Hospital Israelita Albert Einstein, São Paulo, Brazil.

4 Vita-Salute San Raffaele University, Milan, Italy.

Correspondence: landoni.giovanni@hsr.it

\section{References}

1 Zangrillo A, Beretta L, Silvani P, et al. Fast reshaping of intensive care unit facilities in a large metropolitan hospital in Milan, Italy: facing the COVID-19 pandemic emergency. Crit Care Resusc 2020; 22: 91-4.

2 Sartini C, Tresoldi M, Scarpellini P, et al. Respiratory parameters in patients with COVID-19 after using noninvasive ventilation in prone position outside the intensive care unit. JAMA 2020; 15; 323: 2338-40.

3 Zhang $\mathrm{H}$, Penninger JM, Li Y, Zhong N, Slutsky AS. Angiotensinconverting enzyme 2 (ACE2) as a SARS-CoV-2 receptor: molecular mechanisms and potential therapeutic target. Intensive Care Med 2020; 46: 586-90.

4 Khanna A, English SW, Wang XS, et al. ATHOS-3 Investigators. Angiotensin II for the treatment of vasodilatory shock. N Engl J Med 2017; 377: 419-430.

5 Busse LW, Chow JH, McCurdy MT, Khanna AK. COVID-19 and the RAAS-a potential role for angiotensin II? Crit Care 2020; 24: 136.

6 Zangrillo A, Beretta L, Scandroglio AM, et al. Characteristics, treatment, outcomes and cause of death of invasively ventilated patients with COVID-19 ARDS in Milan, Italy. Crit Care ResusC 2020; 22: 200-11.

7 Zangrillo A, Landoni G, Beretta L, et al. Angiotensin II infusion in COVID19-associated vasodilatory shock: A case series. Crit Care 2020; 24: 227.

8 Cavalli G, De Luca G, Campochiaro C, et al. Interleukin 1 blockade with high dose intravenous anakinra in patients with COVID-19, acute respiratory distress syndrome, and hyperinflammation: a retrospective cohort study. Lancet Rheumatol 2020; 2: e325-31.

9 Cabrini L, Idone C, Colombo S, et al. Medical emergency team and non-invasive ventilation outside ICU for acute respiratory failure. Intensive Care Med 2009; 35: 339-43.

10 Hallengren M, Åstrand P, Eksborg S, et al. Septic shock and the use of norepinephrine in an intermediate care unit: Mortality and adverse events. PLOS One 2017; 12: e0183073. 